\title{
THE CENTERS OF SEMI-SIMPLE ALGEBRAS OVER A COMMUTATIVE RING
}

\author{
SHIZUO ENDO and YUTAKA WATANABE
}

Recently A. Hattori introduced in [7], [8], [9] the notion of simple algebras over a commutative ring. Especially, in [9], he examined, as a fundamental problem on simple algebras, whether a directly indecomposable semi-simple algebra is simple or not and gave affirmative answers to this in some particular cases. In this note we shall first prove, as a complete answer to this, that any directly indecomposable semi-simple algebra over a Noetherian ring is simple.

Hattori proposed in [8] several problems on semi-simple algebras. Secondly we shall give some informations on the problem ([8], Problem 4) whether a central semi-simple algebra is separable or not. Furthermore we shall show a class of $p$-trivial simple algebras which is different from that in [11] ([8], Problem 11), and finally we shall give the commutor theory of simple subalgebras in a central separable algebra in the complete form.

Throughout this note we denote by $R$ a commutative ring and by $A$ a not always commutative ring.

A semi-simple $R$-algebra $A$ is said to be a simple $R$-algebra (cf. [9], [11]), if there exists a left $\Lambda$-module $E$ satisfying the following conditions:

i) $E$ is a finitely generated projective $\Lambda$-module.

ii) $E$ is $\Lambda$-indecomposable.

iii) $E$ is $\Lambda$-completely faithful.

By [5], (6. 1), if $\Lambda$ is finitely generated over its center, we can replace iii) by the following iii') $E$ is $\Lambda$-faithful.

1. Decomposability of a semi-simple algebra to simple algebras. We begin with

Received August 18, 1966.

This work was supported by the Matsunaga Science Foundation. 
Lemma 1.1. Let $\Lambda$ be an $R$-algebra which is a finitely generated $R$-module and $P$ a finitely generated projective left (right) A-module, Then

(1) $\mathfrak{T}_{\Lambda}(P)$ is a finitely generated $R$-module. ${ }^{(*)}$

(2) For any multiplicative system $S(\nexists 0)$ of $R$, we have

$$
\mathfrak{I}_{A_{s}}\left(P_{s}\right)=\left\{\mathfrak{I}_{\Lambda}(P)\right\}_{s}
$$

Proof. As it is easy, we omit it.

Proposition 1.2. Let $\Lambda$ be a ring with center $C$. Suppose that $\Lambda$ is a finitely generated $C$-module and that, for any maximal ideal $\mathfrak{m}$ of $C, \Lambda / \mathfrak{m} \Lambda$ is a primary ring. Then the following statements are equivalent:

(1) $C$ is directly indecomposable.

(2) Any finitely generated projective non-zero left (right) A-module is A-completely faithful.

(3) There exists a finitely generated, projective, completely faithful, indecomposable left (right) A-module.

Proof. The implication (3) $\Longrightarrow(1)$ is obvious. Suppose that $C$ is directly indecomposable. Let $P$ be a finitely generated projective non-zero left (right) $\Lambda$-module. By (1.1) and [5], (1.2), $\mathfrak{T}_{\Lambda}(P)$ is an idempotent two-sided ideal of $A$ which is a finitely generated $C$-module. Let $\mathfrak{M}$ be a maximal two-sided ideal of $\Lambda$, and put $\mathfrak{m}=\mathfrak{M} \cap C$. Then $\mathfrak{m}$ is a maximal ideal of $C$ and we have $\mathfrak{M}^{l} \subseteq \mathfrak{m} \Lambda$ for some positive integer $l$. If $\mathfrak{T}_{\Lambda}(P) \subseteq \mathfrak{M}$, then we have $\mathfrak{I}_{\Lambda}(P) \subseteq \mathfrak{m} \Lambda$ (since $\mathfrak{T}_{\Lambda}(P)$ is an idempotent ideal of $\Lambda$ ). According to (1.1), we have $\mathfrak{I}_{\Lambda_{\mathfrak{m}}}\left(P_{\mathfrak{m}}\right)=\left\{\mathfrak{T}_{\Lambda}(P)\right\}_{\mathfrak{m}} \subseteq \mathfrak{m} \Lambda_{\mathfrak{m}}$. Hence we have $\mathfrak{m} P_{\mathfrak{m}}=P_{\mathfrak{m}}$ and so we obtain $P_{\mathfrak{m}}=0$. As $\mathfrak{T}_{\Lambda}(P)$ is $C$-finitely generated, we have $s \mathfrak{T}_{\Lambda}(P)=0$ for some $s$ in $C-\mathfrak{m}$. Now we put $\mathfrak{c}=\operatorname{Ann}_{C} \mathfrak{T}_{\Lambda}(P)$. Then, if $\mathfrak{T}_{\Lambda}(P) \subseteq \mathfrak{m} \Lambda$, we have $\mathfrak{c} \nsubseteq \mathfrak{m} \Lambda$. Hence a twosided ideal $\mathfrak{c} A+\mathfrak{T}_{\Lambda}(P)$ is not contained in any maximal two-sided ideal of $\Lambda$. Therefore we have $\Lambda=\mathfrak{c} A+\mathfrak{T}_{\Lambda}(P) . \quad$ As $\mathfrak{c} \mathfrak{T}_{\Lambda}(P)=0, \Lambda$ is the direct sum of twosided ideals $\mathfrak{c} A$ and $\mathfrak{T}_{\Lambda}(P)$. Since $C$ is directly indecomposable and $\mathfrak{I}_{\Lambda}(P) \neq 0$, we have $\mathfrak{I}_{\Lambda}(P)=\Lambda$. This proves $(1) \Longrightarrow(2)$.

Suppose (2), and let $e$ be a non-trivial idempotent of $A$. Then $A e$ is $A$ completely faithful and therefore we have $\Lambda e \Lambda=\Lambda$. Hence $e$ is not contained in any $\mathfrak{m} \Lambda$. Therefore the image of $e$ in $\Lambda / \mathfrak{m} \Lambda$ is also a non-trivial idempotent of $\Lambda / \mathfrak{m} \Lambda$. Since $\Lambda / \mathfrak{m} \Lambda$ is an Artinian primary ring, there are a finite number of

(*) $\mathfrak{T}_{\Lambda}(P)$ denotes the trace ideal of $P$ (cf. [1]). 
orthogonal primitive idempotents in $\Lambda / \mathfrak{m} \Lambda$. Then there are also only a finite number of orthogonal primitive idempotents in $A$. This implies (2) $\Longrightarrow(3)$.

The implications $(1) \Leftrightarrow(3)$ in the following theorem were proved in [9], Theorem 4 in case $C$ is Noetherian.

Theorem 1. 3. Let $\Lambda$ be a separable $R$-algebra and $C$ the center of $\Lambda$. Then the following conditions are equivalent:

(1) $C$ is directly indecomposable

(2) Any finitely generated projective non-zero left (right) A-module is A-completely faithful.

(3) $\Lambda$ is a simple algebra.

Proof. It suffices to show that $A$ satisfies the assumptions in (1.2). However, as $\Lambda$ is a separable $R$-algebra, $A$ is a finitely generated $C$-module by [2], (1.2) and $\Lambda / \mathfrak{m} \Lambda$ is a central separable $C / \mathfrak{m} C$-algebra for any maximal ideal $\mathfrak{m}$ of $C$ by [2], (1. 4). This completes our proof.

Lemma 1. 4. Let $R$ be a complete local ring with a maximal ideal $\mathfrak{m}$ and $\Lambda$ be an $R$-algebra which is a finitely generated $R$-module. Then $A$ is directly indecomposable if and only if $\Lambda / \mathfrak{m} \Lambda$ is so.

Proof. We have only to prove the only if part. Let $\bar{e}$ be a central idempotent of $\Lambda / \mathfrak{m} \Lambda$. As $R$ is complete, there exists an idempotent $e$ of $\Lambda$ whose image in $\Lambda / \mathfrak{m} \Lambda$ coincides with $\bar{e}$. Now it suffices to show that $e$ is central. Since $e$ is central in $\Lambda / \mathfrak{m} \Lambda$, we have $\lambda e-e \lambda \in \mathfrak{m} \Lambda$ for any $\lambda$ of $\Lambda$. Then we have $e \lambda e-e \lambda, \lambda e-e \lambda e \in \mathfrak{m} \Lambda$. If we put $e \lambda e-e \lambda=\sum_{i=1}^{t} m_{i} \lambda_{i}, m_{i} \in \mathfrak{m}, \lambda_{i} \in \Lambda$, then we have $e \lambda e-e \lambda=e(e \lambda e-e \lambda)-(e \lambda e-e \lambda) e=\sum_{i=1}^{t} m_{i}\left(e \lambda_{i}-\lambda_{i} e\right) \in \mathfrak{m}^{2} \Lambda . \quad$ By repeating the same procedure, we see $e \lambda e-e \lambda \in \mathfrak{m}^{l} \Lambda$, for any $l>0$. As $\bigcap_{l=1}^{\infty} \mathfrak{m}^{l} \Lambda=0$, we obtain $e \lambda e=e \lambda$. Similarly we can show $e \lambda e=\lambda e$. So $\lambda e=e \lambda$ for any $\lambda$ of $\Lambda$, which completes our proof.

Lemma 1.5. Let $A$ be a central $R$-algebra which is a finitely generated $R$-module, and $S$ be a commutative $R$-algebra which is a flat $R$-module. Then $S \otimes_{R} \Lambda$ is a central S-algebra.

Proof. See [6], Chap. V, 6, Lemma 3.

Now we give, as our main result, the following 
Theorem 1. 6. Let $R$ be a Noetherian ring and $A$ a semi-simple $R$-algebra which is a finitely generated $R$-module. Let $C$ be the center of $\Lambda$.

Then the following conditions are equivalent:

(1) $C$ is directly indecomposable.

(2) Any finitely generated projective non-zero left (right) 1-module is 1-completely faithful.

(3) $\Lambda$ is a simple algebra.

So, a semi-simple algebra over a Noetherian ring $R$, which is a finitely generated $R$ module, is expressible as the direct sum of a finite number of simple $R$-algebras.

Proof. It suffices to show that $\Lambda$ satisfies the assumptions in (1.2). As $\Lambda$ is a central semi-simple $C$-algebra, $A_{\mathfrak{m}}$ is also a central semi-simple $C_{\mathfrak{m}}$-algebra for any maximal ideal $\mathfrak{m}$ of $C$ by $(1.5)$. Let $\hat{C}_{\mathfrak{m}}$ be the completion of $C_{\mathfrak{m}}$. and put $\hat{\Lambda}_{\mathfrak{m}}=\hat{C}_{\mathfrak{m}} \otimes \Lambda$. Then, again by $(1.5), \hat{\Lambda}_{\mathfrak{m}}$ has no non-trivial central idempotent, and then, by (1. 4), $\hat{\Lambda}_{\mathfrak{m}} / \mathfrak{m} \hat{\Lambda}_{\mathfrak{m}}$ also has no non-trivial central idempotent.

Since $\Lambda / \mathfrak{m} \Lambda$ is semi-simple and we have $\Lambda / \mathfrak{m} \Lambda \cong \hat{\Lambda}_{\mathfrak{m}} / \hat{\Lambda}_{\mathfrak{m}} \cong \Lambda_{\mathfrak{m}} / \mathfrak{m} \Lambda \mathfrak{m}, \Lambda / \mathfrak{m} \Lambda$ must be simple. This completes our proof.

\section{Separability of a central semi-simple algebra.}

We first give

Proposition 2. 1. Let $R$ be an Artinian ring and $\Lambda$ be a central semi-simple algebra over $R$ which is a finitely generated $R$-module. Then the following conditions are equivalent:

(1) $\Lambda$ is a separable algebra.

(2) $\Lambda$ is a projective $R$-module.

(3) $\Lambda$ is a completely faithful R-module.

Proof. The implications (1) $\Longrightarrow(2) \Longrightarrow(3)$ of our theorem follow from [2], (1. 2) and [1], (A. 3). Hence we have only to prove (3) $\Longrightarrow(1)$. Without loss of generality we may assume, according to (1.6), that $R$ is a local ring with a maximal ideal $\mathfrak{m}$ and that $\Lambda$ is a central simple $R$-algebra with a unique maximal two-sided ideal $\mathfrak{m} \Lambda$. Suppose that $\Lambda$ is a completely faithful $R$ module. Then we have $A=R \oplus M$ for an $R$-module $M$. Let $\bar{\alpha}$ be an element of the center of $\Lambda / \mathfrak{m} \Lambda$ and $\alpha$ a representative of $\bar{\alpha}$ in $\Lambda$. Then we have $\alpha \lambda-\lambda \alpha \in \mathfrak{m} \Lambda$ for any $\lambda$ of $\Lambda$. Let $l$ be a non-negative integer such that $\mathfrak{m}^{l} \neq 0$ 
but $\mathfrak{m}^{l+1}=0$.

Then we have $\mathfrak{m}^{l}(\alpha \lambda-\lambda \alpha)=0$ for any $\lambda$ of $\Lambda$. As $R$ is the center of $\Lambda$, we have $\mathfrak{m}^{l} \alpha \subseteq R$. Now put $\alpha=r+u, r \in R, u \in M$. Then, as $\Lambda=R \oplus M$ as $R$-modules, we have $\mathfrak{m}^{l} u=0$. Since $\mathfrak{m} \Lambda$ is a unique maximal two-sided ideal of $\Lambda$, we have $u=\alpha-r \in \mathfrak{m} \Lambda$, and so we have $\bar{\alpha}=\bar{r} \in R / \mathfrak{m} \subseteq \Lambda / \mathfrak{m} \Lambda$. This proves that $\Lambda / \mathfrak{m} \Lambda$ is a central simple $R / \mathfrak{m}$-algebra. By virtue of the classical result, $\Lambda / \mathfrak{m} \Lambda$ is a separable $R / \mathfrak{m t}$-algebra. According to [2], (4.7), $A$ is also a separable $R$ algebra. This proves the implication $(3) \Longrightarrow(1)$.

Corollary 2. 2. Let $R$ be a (quasi-) Frobenius ring and $A$ a central semisimple $R$-algebra which is a finitely generated $R$-module. Then $\Lambda$ is a separable $R$ algebra and $\Lambda$ is also a Frobenius ring.

Proof. As $R$ is Frobenius, $A$ is $R$-completely faithful, as is well known. By (2. 1) $\Lambda$ is a separable $R$-algebra, and is a projective $R$-module. Then, according to [5], (3.6), $\Lambda$ is a Frobenius $R$-algebra, and so $\Lambda$ is a Frobenius ring.

This corollary is an affirmative answer, in case $R$ is Frobenius, to the following

Рвовкем H. Is any central semi-simple R-algebra (which is a finitely generated $R$-module) a separable $R$-algebra?

Theorem 2. 3. If the answer to Problem $H$ is affirmative for any Artinian ring $R$, then it is also affirmative for any Noetherian ring $R$.

Proof. Let $R$ be a Noetherian ring and $\Lambda$ a central semi-simple $R$ algebra. Now it suffices, by [2], (4.7), to show that, for any maximal ideal $\mathfrak{m}$ of $R, \Lambda / \mathfrak{m} \Lambda$ is a central simple $R / \mathfrak{m}$-algebra. Hence, by (1.6) we may suppose that $R$ is a complete local ring with a maximal ideal $\mathfrak{m}$ and that $\Lambda$ is a central simple $R$-algebra with a unique maximal two-sided ideal $\mathfrak{m} \Lambda$. Therefore we have only to prove that $\Lambda / \mathfrak{m} \Lambda$ is a central $R / \mathfrak{m}$-algebra.

Let $\bar{C}_{l}$ be the center of $\Lambda / \mathfrak{m}^{l} \Lambda$ for any positive integer $l$. Since $\Lambda / \mathfrak{m}^{l} \Lambda$ is a simple $R / \mathfrak{m}^{l}$-algebra, $\Lambda / \mathfrak{m}^{l} \Lambda$ is a central simple $\bar{C}_{l}$-algebra. As $R / \mathfrak{m}^{l}$ is Artinian, $\bar{C}_{l}$ is also an Artinian local ring. If the answer to Problem $\mathrm{H}$ is affirmative for Artinian rings, then $\Lambda / \mathfrak{m}^{l} \Lambda$ is a central separable $\bar{C}_{l}$-algebra. Then $\mathfrak{m} \bar{C}_{l}$ is a maximal ideal of $\bar{C}_{l}$ and we have $\mathfrak{m}^{l} \bar{C}_{l+1}=\mathfrak{m}^{l}\left(\Lambda / \mathfrak{m}^{l+1} \Lambda\right) \cap \bar{C}_{l+1}$. By [2], (1. 4), we have $\bar{C}_{l}=\bar{C}_{l+1} / \mathfrak{m}^{l} \bar{C}_{l+1}$. If we put $C=\lim \bar{C}_{l}$, we have $C \subseteq \Lambda$ as $R$ is complete. Let $\alpha$ be an element of $C$. Then, for any positive integer $l$ we have $\alpha \lambda-\lambda \alpha \in$ 
$\mathfrak{m}^{l} C$ for any $\lambda$ of $\Lambda$, and so we have $\alpha \lambda-\lambda \alpha=0$ as $\bigcap_{l=1}^{\infty} \mathfrak{m}^{l} \Lambda=0$. Hence we have $\alpha \in R$, which proves $C \subseteq R$. Since for any $l, C \rightarrow \bar{C}_{l}$ is an epimorphism induced by $\Lambda \rightarrow \Lambda / \mathfrak{m}^{l} \Lambda$, we have $\bar{C}_{l}=C / \mathfrak{m}^{l} \Lambda \cap C$, and, especially we have $\bar{C}_{1}=C / \mathfrak{m} \Lambda \cap C$ $\subseteq R / \mathfrak{m} \Lambda \cap R=R / \mathfrak{m}$. Thus we see $\bar{C}_{1}=R / \mathfrak{m}$. This shows that $\Lambda / \mathfrak{m} \Lambda$ has $R / \mathfrak{m}$ as its center, which completes our proof.

\section{3. p-trivial simple algebras.}

We see that a simple algebra $\Lambda$ over a Noetherian ring $R$ is $p$-trivial ([4]) if and only if there is only one division algebra to which $\Lambda$ belongs. In [11] it was shown that any simple algebra over a complete local ring is $p$-trivial (In [11], $p$-trivial algebras are called 'strongly simple' algebras). However, this can not be generalized to simple algebras over a non-complete local ring without further assumptions. In this section we shall give another class of $p$ trivial simple algebras.

Proposition 3. 1. Let $R$ be a Noetherian integrally closed integral domain and $\Lambda a$ simple $R$-algebra which is a finitely generated projective $R$-module. Then the center of $\Lambda$ is also a Noetherian integrally closed integral domain.

Proof. Let $C$ be the center of $A$. Then $C$ is an indecomposable Noetherian ring. Let $K$ be the quotient field of $R$ and put $\Sigma=K \bigotimes_{R} \Lambda$. Then $\Sigma$ is a semi-simple $K$-algebra. Let $e$ be a central idempotent of $\Sigma$. As $\Lambda$ is a simple $R$-algebra, $\Lambda_{\mathfrak{p}}$ is a semi-simple $R_{\mathfrak{p}}$-algebra for any prime ideal $\mathfrak{p}$ of height 1 in $R$. Since $R_{\mathfrak{p}}$ is a discrete valuation ring, $\Lambda_{\mathfrak{p}}$ is a maximal $R_{\mathfrak{p}}$-order in $\Sigma$ and $C_{\rho}$ is a hereditary ring (cf. [7], [9]). Therefore we have $e \in C_{\mathrm{p}}$. As $A$ is a finitely generated projective $R$-module, we have $\Lambda=\bigcap_{h t p=1} \Lambda_{p}$, and, from this we obtain $C=\bigcap_{h t p=1} C_{p}$. Hence we must have $e \in C$. Since $C$ is directly indecomposable, this shows that $\Sigma$ is a simple $K$-algebra. Consequently $C$ must be an integral domain. Since each $C_{p}$ is hereditary, $C$ is also integrally closed.

Lemмa 3.2. Let $R$ be a Noetherian local integral domain and $K$ the quotient field of $R$. Let $\Lambda$ be a simple $R$-algebra which is a finitely generated projective $R$-module such that $K \underset{R}{\otimes} \Lambda$ is a division $K$-algebra. Then any finitely generated projective $\Lambda$-module is free.

Proof. This follows immediately from [3], Theorem 2, since $\Lambda / \mathfrak{m} \Lambda$ is a semi-simple $R / \mathfrak{m}$-algebra for a maximal ideal $\mathfrak{m}$ of $R$. 
Theorem 3. 3. Let $R$ be a semi-local regular domain with Krull dimension $\leqq 2$. Then any simple $R$-algebra $\Lambda$, which is a finitely generated projective $R$-module, is $p$-trivial.

Proof. Let $K$ be the quotient field of $R$. Then, by (3.1), $K \underset{R}{\otimes} \Lambda$ is a simple $K$-algebra. Let $e$ be a primitive idempotent of $K \otimes \Lambda$ and put $\mathfrak{l}=\left(K \otimes_{R} \Lambda\right) e \cap \Lambda . \quad$ Then $\Lambda / \mathfrak{I}$ is a torsion-free $R$-module and we have $K \underset{R}{\otimes} \mathfrak{l}=$ $(K \underset{R}{\otimes} \Lambda)$ e. Since gl. $\operatorname{dim} \Lambda \leqq \mathrm{gl} \cdot \operatorname{dim} R \leqq 2$, we have $\operatorname{dh}_{\Lambda} \Lambda / \mathfrak{l} \leqq 1$, and so $\mathfrak{l}$ is: projective. $\mathfrak{l}$ is obviously $\Lambda$-faithful, so it is also $\Lambda$-completely faithful. Now put $\Omega=\operatorname{End}_{\Lambda}(\mathfrak{l})$. Then $K \underset{R}{\otimes} \Omega$ is a division $K$-algebra and $\Omega$ is a simple (division) $R$-algebra which is Morita-equivalent to $\Lambda$. According to (3.2), any finitely generated projective $\Omega_{\mathfrak{m}}$-module is free for any maximal ideal $\mathfrak{m}$ of $R$. As $R$ is semi-local, any finitely generated projective $\Omega$-module is free (cf. [3], Theorem 1), and therefore $\Omega$ is $p$-trivial. Since $\Omega$ is Morita equivalent to $\Lambda, \Lambda$ is also $p$-trivial ([4], [11]).

Remark. There exists a simple algebra over a non-semi-local principal ideal domain which is not $p$-trivial. Accordingly, Theorem 3.3 can not be generalized to a non-semi-local principal ideal domain.

\section{Tensor products and Commutor theory.}

This section is concerned with the commutor theory of a central separable algebra and its simple subalgebras.

First we give

Proposition 4. 1. Let $\Gamma$ be an R-algebra which is a finitely generated projective $R$-module and $\Lambda$ be a semi-simple $R$-subalgebra of $\Gamma$. Then $\Lambda$ is a $\Lambda$-direct summand of $\Gamma$.

Proof. By the semi-simplicity of $\Lambda$ and $R$-projectivity of $\Gamma, \Gamma$ is a $\Lambda$ projective and (clearly) $\Lambda$-faithful $\Lambda$-module, so a completely faithful $\Lambda$-module by [5], (6.1). Then $\Lambda$ is a $\Lambda$-direct summand of $I$.

A semi-simple subalgebras of a central separable algebra splits by the above proposition. So, the coherent condition of the Hattori's commutor theory ([7]) is always satisfied. Then we can give, as a supplement to a Hattori's theorem, the following

Theorem 4.2 ([7]). Let $\Gamma$ be a central separable algebra over a commutative ring $R$, and $\Lambda$ be a semi-simple $R$-subalgebra. Then: 
(1) $V_{\Gamma}(\Lambda)$ is semi-simple. ${ }^{(*)}$

(2) $V_{\Gamma}\left(V_{\Gamma}(\Lambda)\right)=\Lambda$.

(3) $V_{\Gamma}(\Lambda)$ is Morita-equivalent to $\Gamma \underset{R}{\otimes} \Lambda^{0}$ where $\Lambda^{0}$ denotes the opposite algebra of $\Lambda$.

Lemma 4. 3. Let $\Gamma$ be a central $R$-algebra having $R$ as an $R$-direct summand, and $\Lambda$ be an R-projective algebra. Then the center of $\Lambda \otimes_{R} \Gamma$ coincides with the center of $\Lambda$.

Proof. At first, we assume that $R$ is a local ring. Since $\Lambda$ is free, any element of $\Lambda \otimes_{R} \Gamma$ is uniquely expressed by the form $\sum u_{i} \otimes \gamma_{i}$, where $\left\{u_{i}\right\}$ is a basis of $\Lambda$ and $\gamma_{i} \in \Gamma$. If $z=\sum u_{i} \otimes \gamma_{i}$ lies in the center of $\Lambda \otimes_{R} \Gamma, z$ commutes with all $1 \otimes \gamma$, so $\sum u_{i} \otimes\left(\gamma_{i} \gamma-\gamma \gamma_{i}\right)=0$. Then we get $\gamma_{i} \in$ the center of $\Gamma=R$ (i.e. $\left.z=\sum \gamma_{i} u_{i} \otimes 1\right)$. Therefore we get $\sum\left(\left(\gamma_{i} u_{i}\right) \lambda-\lambda\left(\gamma_{i} u_{i}\right)\right) \otimes 1=0$ for any $\lambda \in \Lambda$, so $\sum \gamma_{i} u_{i} \lambda=\lambda \Sigma \gamma_{i} u_{i}$ by the assumption of Lemma. Hence $z$ is in the center of A. The converse inclusion is trivial.

In the case that $R$ is global, for any maximal ideal $\mathfrak{m}$ of $R, \Lambda_{\mathfrak{m}}$ is $R_{\mathfrak{m}}$ projective. $\Gamma_{\mathfrak{m}}$ is a central $R_{\mathfrak{m}}$-algebra and $R_{\mathfrak{m}}$ is an $R_{\mathfrak{m}}$-direct summand of $\Gamma_{\mathrm{m}}$.

Let $z$ be in the center of $\Lambda \underset{R}{\otimes_{R}} \Gamma$. Then $Z$ is in the center of $\Lambda_{\mathrm{m}}$. So there exists $s \notin \mathfrak{m}$ (depending on $\lambda, \mathfrak{m}$ ) such that $s z$ is contained in the center of $\Lambda$. Put $\mathfrak{c}=\{r \in R \mid r z$ is contained in the center of $\Lambda\}$. Then $\mathfrak{c}$ is an ideal of $R$ which is not contained in any maximal ideal. So $1 \in \mathfrak{c}$. Hence $z$ is in the center of $A$.

Proposition 4. 4. Let $R$ be a commutative Noetherian ring. If $\Gamma$ is a central separable $R$-algebra and $\Lambda$ is a simple $R$-algebra which is a finitely generated $R$-projective module, then $\Lambda \otimes_{R} \Gamma$ is a simple $R$-algebra.

Proof. $\Lambda \underset{R}{\otimes} \Gamma$ is semi-simple by [7], (2. 4). Since $R$ is Noetherian, by virtue of (1.6), we have only to show the indecomposability of the center of $\Lambda \underset{R}{\otimes} \Gamma$. But it is an immediate consequence of (4.3).

Let $\Lambda$ be a simple subalgebra of a central separable algebra over a commutative Noetherian ring $R$. Since $\Lambda$ is a $\Lambda$-direct summand of $\Gamma, V_{\Gamma}(\Lambda)$ is Morita-

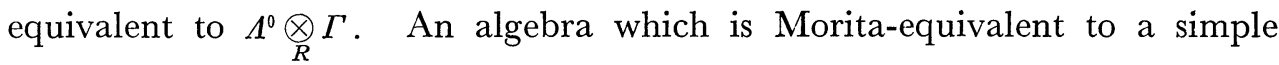
algebra is also simple ([11]), so we get the following

Theorem 4. 5. Let $\Gamma$ be a central separable algebra over a commutative Noetherian ring $R$, and $\Lambda$ be a simple subalgebra of $\Gamma$.

\footnotetext{
(*) $V \Gamma(\Lambda)=\{\gamma \in \Gamma / \gamma \lambda=\lambda \gamma$, for any $\lambda \in \Lambda\}$.
} 
Then,

(1) $V_{\Gamma}(\Lambda)$ is a simple algebra.

(2) $V_{\Gamma}\left(V_{\Gamma}(\Lambda)\right)=\Lambda$.

(3) $\Lambda^{0} \otimes_{R} \Gamma$ is simple and is Morita-equivalent to $V_{\Gamma}(\Lambda)$.

Remark. In Theorem 4. 5, the simplicity of $V_{\Gamma}(\Lambda)$ can be proved by a more simple argument. That is: the center of $V_{\Gamma}(\Lambda)$ contains the center of $\Lambda$, and the center of $V_{\Gamma}\left(V_{\Gamma}(\Lambda)\right)(=\Lambda)$ contains the center of $V_{\Gamma}(\Lambda)$, then the center of $V_{\Gamma}(\Lambda)$ coincides with the center of $\Lambda$. Hence $V_{\Gamma}(\Lambda)$ is simple whenever $\Lambda$ is so.

By virtue of this remark, Proposition 4.4 in the case that $\Lambda$ is a simple subalgebra of $\Gamma$ is proved without help of Lemma 4.3.

\section{REFERENCES}

[ 1 ] M. Auslander and O. Goldman, Maximal orders, Trans. Amer. Math. Soc., 97 (1960), $1-24$.

[2] (1960), 367-409.

[ 3 ] H. Bass, Projective modules over algebras, Ann. of Math., 73 (1963), 532-542.

[4] P.M. Cohn, A remark on matrix rings over free ideal rings, Proc. Camb. Phil. Soc., 62 (1966), 1-4.

[5] S. Endo, Completely faithful modules and quasi-Frobenius algebras, To appear.

[6] P. Gabriel, Des catégories abéliennes, Bull. Soc. Math. France, 90 (1962), 323-428.

[ 7 ] A. Hattori, Semisimple algebras over a commutative ring, J. Math. Soc. Japan, 15(1963), 404-419.

[8] - Semisimple algebras over a commutative ring, Proc. Symp. Algebra. Math. Soc. Japan, 6 (1965), 37-40 (In Japanese).

[9] - Simple algebras over a commutative ring, To appear.

[10] T. Kanzaki, On commutor rings and Galois theory of separable algebras, Osaka Math. J., 1 (1964), 103-115.

[11] Y. Watanabe, Simple algebras over a complete local ring, Osaka Math. J., 3 (1966), 13-20.

Added in proof. Lemma 1.4 holds for a Henselian local ring R. So we can prove the first part of Theorem 1.6 without the assumption that $\mathrm{R}$ is Noetherian, by using the Henselization instead of the completion, Then Theorem 1.3 is a special case of Theorem 1.6. Also we can omit this assumption from Proposition 4.4 and Theorem 4.5. (For Henselian rings see M. Nagata, 'Local rings', Interscience, 1962).

Tokyo University of Education 